\title{
A resiliência em trabalhadores da área da enfermagem?
}

\author{
The resilience of workers in nursing
}

\author{
Maria de Fátima BELANCIERI ${ }^{2}$ \\ Marli Luiz BELUCl ${ }^{3}$ \\ Daniela Vitti Ribeiro da SILVA ${ }^{4}$ \\ Ederli Aparecida GASPARELO ${ }^{5}$
}

\section{Resumo}

O objetivo deste estudo foi investigar o nível de resiliência dos trabalhadores de enfermagem, visando ao conhecimento das fraquezas e fortalezas desse profissional diante das adversidades a que está submetido. Participaram enfermeiros e auxiliares de enfermagem da rede pública de saúde. Para a coleta de dados, utilizaram-se ficha de dados sociodemográficos e o Questionário do Coeficiente de Resiliência, com metodologia quantitativa. A maioria dos participantes, em relação ao fator regulação de emoções, está abaixo da média, demonstrando que a capacidade de resiliência nesse fator encontra-se enfraquecida. No fator controle de impulsos, a maioria está acima da média. Nos outros fatores (otimismo, análise causal, empatia, autoeficácia e exposição), a maioria se apresenta na média. O excessivo controle de impulsos e a dificuldade na regulação das emoções acarretam grande dispêndio de energia por parte do trabalhador, uma vez que ele não pode exteriorizar suas emoções, especialmente no ambiente de trabalho, justificando o alto índice de estresse entre os enfermeiros.

Unitermos: Enfermagem. Psicologia. Resiliência, psocilógica.

\begin{abstract}
This study aimed to investigate the resilience level of nursing workers, in order to find out the strengths and weaknesses of the worker in the face of the adversities that may present themselves. Nurses and auxiliary nurses in the Public Health Service participated. To collect data, socio-demographic data records and the coefficient Resilience Questionnaire wereemployed, using quantitative methodology. With regard to the Regulation of Emotions factor, most participants are below average, demonstrating that the capacity for resilience, in this factor, is weakened. With the Control of Impulses factor, most are above average. For the other factors (Optimism, Causal Analysis, Empathy, Self-efficacy and Exhibition) the majority are average. The excessive Control of Impulses and the difficulty in regulating the emotions involve a large expenditure of energy on the part of the worker since they cannot be externalized, especially in the workplace, thereby explaining the high rate of stress among nurses.
\end{abstract}

Uniterms: Nursing. Psychology. Resilience, psychological.

QVR

1 Estudo vinculado ao Grupo de Estudos e Pesquisa em Psicologia Hospitalar. Universidade Sagrado Coração. Colaboraram no estudo: Verônica Lima dos Reis, Ana Beatriz Sacomano Montassier, Ana Vera Niquerito, Nilseia Meneguel Coltro, Marcella Carvalho Martins.

2 Universidade Sagrado Coração, Curso de Psicologia, Programa de Pós-Graduação. R. Ir. Arminda, 10-50, 17011-160, Bauru, SP, Brasil. Correspondência para/Correspondence to: M.F. BELANCIEREI. E-mail: <mfbelancieri@grupoguidiotrujillo.com>.

3 Universidade de São Paulo, Programa de Pós-Graduação em Ciências da Reabilitação, Hospital de Reabilitação de Anomalias Craniofaciais. Bauru, SP, Brasil.

4 Universidade Sagrado Coração, Grupo de Estudos e Pesquisa em Psicologia Hospitalar. Bauru, SP, Brasil.

5 Universidade Sagrado Coração, Curso de Psicologia. Bauru, SP, Brasil. 
A enfermagem é parte integrante e fundamental nas equipes de saúde e constitui-se o maior grupo de trabalhadores na área. É responsável por 60\% das ações, prestando assistência 24 horas por dia, implementando e cuidando da saúde da população, visando à promoção, à prevenção, à manutenção e à recuperação da saúde. É a categoria que mais contato tem com o ambiente de saúde e com os doentes, num clima de dor e sofrimento que geralmente emerge nesse contexto (Bulhões, 1994).

Belancieri (2003; 2005; 2007) ressalta em seus estudos que o ambiente de trabalho da enfermagem constitui-se de inúmeros elementos estressores, o que pode comprometer a saúde e a qualidade de vida dos trabalhadores. No setor público, Stacciarini e Tróccoli (2001) afirmam que a enfermagem é classificada pela Health Education Authority como a quarta profissão mais estressante.

Estudo realizado por Belancieri $(2003 ; 2005)$ com 300 trabalhadores da área da enfermagem, visando à identificação dos fatores e dos níveis de stress, bem como dos transtornos psicossomáticos autoatribuídos, evidenciou os seguintes resultados: 100\% dos trabaIhadores participantes do estudo apresentavam estresse em algum nível; em relação aos fatores desencadeadores de estresse, observou-se o controle excessivo por parte da instituição; dificuldades nas relações interpessoais; inobservância da ética pelos colegas; atividades rotineiras e repetitivas; excessivo número de pacientes; clima de sofrimento e morte; salários insuficientes; falta de lazer; falta de apoio e reconhecimento pela instituição, entre outros. Os sintomas psicossomáticos predominantes foram cansaço, tensão muscular, nervosismo, irritabilidade, dor lombar, ansiedade, tensão pré-menstrual, cefaleias, problemas de memória, depressão, entre outros.

Diante desses estudos, observamos que os trabaIhadores da área da enfermagem sofrem forte impacto de estressores internos e externos, podendo comprometer sua saúde e sua qualidade de vida, bem como afetar seu desempenho profissional, colocando em risco a assistência aos usuários do sistema de saúde. Nesse sentido, torna-se relevante a investigação do nível de resiliência, visando ao conhecimento das fraquezas e fortalezas do trabalhador diante das adversidades a que está submetido. Desse modo, será possível implementar 228 programas que visem a fortalecer as estratégias de enfrentamento diante das adversidades que experienciam em seu cotidiano pessoal ou profissional.

\section{Resiliência entre os trabalhadores da área da enfermagem}

Resiliência é um termo relativamente novo na área da saúde. Historicamente, surge associado aos estudos da Física e da Engenharia, referindo-se à capacidade que um material tem de absorver energia sem sofrer deformações permanentes (Yunes \& Szimanski, 2001). No sentido original, pressupõe uma resistência do material que, ao sofrer um impacto ou choque com outros materiais, não perde suas propriedades.

As autoras revelam que o termo resiliência aparece em contraposição aos termos invencibilidade e invulnerabilidade que apresentam características de resistência absoluta ao estresse. Yunes (2001) ressalta que a resiliência consiste na habilidade de superar as adversidades, o que não significa que o indivíduo saia da crise ileso, como sugere os termos invulnerabilidade e invencibilidade.

Para Pereira (2001), a resiliência consiste na capacidade universal que permite ao indivíduo, grupo ou comunidade prevenir, minimizar ou ultrapassar as marcas ou efeitos das adversidades.

A noção de resiliência pertence à estrutura do desenvolvimento psicológico, segundo Ralha-Simões (2001), podendo ser traduzida como a capacidade pessoal de enfrentar a adversidade, não no sentido de resistir, mas de ultrapassá-la e superá-la com êxito.

Moraes e Rabinovich (1996) revelam que esse processo ocorre em um determinado tempo devido a certas combinações benéficas de atributos individuais, familiares, ambientais, sociais e culturais. Especificamente, as relações que são constituídas nos diversos ambientes são grandes preditores de resiliência (Polleto \&Koller, 2008).

Lindstron (2001) complementa, levando em consideração três aspectos: 1) as características individuais, como a genética, a fase de desenvolvimento, o gênero, a constituição e a experiência e histórico de vida; 2) o contexto, como o suporte social, a classe social, a cultura e o ambiente; 3) a quantidade e qualidade dos eventos de vida, como o desejável, o controlável, a sua 
magnitude, a duração no tempo e efeitos a longo prazo.

A presença de fatores de risco não prediz psicopatologias, no entanto a presença de fatores de proteção é preditiva de resiliência. Assim, "os fatores de proteção promovem, no indivíduo, ganho de controle sobre sua vida e incentivo ao bem-estar, à saúde psicológica, ainda que frente aos fatores de risco" (Trombeta \& Guzzo, 2002, p.32).

Para que o sujeito seja considerado resiliente, é necessário que exista um equilíbrio, ou uma combinação entre os fatores de risco e de proteção. De acordo com as autoras, os fatores de proteção estão associados às condições do próprio indivíduo (autoestima positiva, temperamento fácil e maleável, ou seja, flexibilidade diante das adversidades), às condições familiares (ambiente familiar onde predominem coesão, estabilidade, flexibilidade, adaptabilidade, valores, crenças, etc.; e pais amorosos, competentes, interessados, com participação na vida escolar dos filhos, expectativas positivas em relação ao futuro dos filhos, elogiando seus esforços etc.) e às condições ambientais (comunicação aberta, limites definidos e realistas, tolerância aos conflitos, respeito, reconhecimento e aceitação, receptividade a novas ideias etc.).

Com relação ao polo oposto aos fatores de proteção, ou seja, aos fatores de risco, estão pobreza crônica, temperamento difícil (mau humor, baixa tolerância à frustração, passividade, comportamento destrutivo, autoestima negativa, cinismo e hostilidade, habilidade de comunicação pobre, depressão, tentativa de suicídio e abuso de álcool e drogas), exposição a eventos estressantes ou traumáticos de vida e características ambientais (estruturas rigidamente organizadas ou muito desorganizadas).

Apesar de os fatores de proteção e risco apresentarem características bem definidas e claras, o sujeito que convive com fatores de risco não deixa de ser resiliente, mas a maneira como convive com tais riscos é que orienta a proteção e a possibilidade de ser resiliente diante das adversidades.

Junqueira e Deslandes (2003) ressaltam que essa capacidade pode ser desenvolvida a partir das relações que estabelecem vínculos afetivos e de confiança. Entretanto, desenvolver a resiliência não significa a pessoa superar todas as experiências traumáticas, podendo ser resiliente em algumas situações e não em outras.
Diversos autores descrevem as principais características do sujeito resiliente (Flach, 1997; Grotberg, 2005; Nelson, 1997; Polk, 1997; Reivich \& Shatté, 2002; S.J. Wolin \& S. Wolin, 1993). Nossas análises e discussões, no entanto, estarão fundamentadas especificamente em Reivich e Shatté (2002), autores do instrumento de coleta de dados utilizado neste estudo.

Ao construir o Questionário do Coeficiente de Resiliência (RQ-Test), Reivich e Shatté (2002) organizam a resiliência em sete fatores: Regulação das Emoções; Controle de Impulsos; Otimismo; Análise Causal; Empatia; Autoeficácia e Exposição.

Acredita-se que a resiliência possa ser desenvolvida ao longo da existência, quando o sujeito apropria-se de sua realidade e a transforma, transformando, consequentemente, a si mesmo num movimento dialético (Belancieri, 2007). Assim, conhecer os níveis de resiliência propostos por Reivich e Shatté (2002) poderá auxiliar na elaboração de programas que visem à redução do estresse e à promoção da Resiliência, em que o trabalhador possa construir novos sentidos e ações, buscando a superação e a transformação das condições atuais de saúde e de trabalho a que estão submetidos, visando melhorar sua qualidade de vida.

\section{Método}

Este estudo foi realizado na Rede Básica de Saúde, Serviços de Urgência e Emergência e Unidades e Serviços Especializados, na Região Centro-Oeste do Estado de São Paulo. Participaram do estudo trabalhadores da área da enfermagem (enfermeiros e auxiliares de enfermagem) da rede pública de saúde, contratados por meio de concurso público municipal.

Os instrumentos utilizados foram: ficha de dados sociodemográficos, que visou à coleta dos dados pessoais e profissionais, e o Questionário do Coeficiente de Resiliência-RQ-Test (Reivich \& Shattè, 2002), visando a avaliar os níveis de resiliência.

Para a viabilização do projeto, primeiramente, foi solicitado à Secretaria de Saúde do Município autorização para realização do estudo, bem como o envio ao Comitê de Ética em Pesquisa. Assim, este estudo está em conformidade com as resoluções do Conselho 
Nacional de Saúde (CNS 196/96), que dispõem sobre a realização de investigações com seres humanos, sendo aprovado em 20/3/2007, sob o protocolo no 11/07.

Posteriormente, foi solicitado à Secretaria Municipal de Saúde, um levantamento dos trabalhadores da área da enfermagem contratados na rede municipal de saúde. Foi realizado um primeiro contato com as respectivas unidades, visando discutir os objetivos do projeto e agendar data e horário para a aplicação dos instrumentos de pesquisa, feita individualmente ou em pequenos grupos, conforme as condições do momento.

A avaliação e a interpretação dos dados foram realizadas por meio de procedimentos recomendados pelos autores do instrumento (Reivich \& Shattè, 2002), seguindo uma metodologia quantitativa.

\section{Resultados e Discussão}

Dos 430 trabalhadores da área da enfermagem da rede pública de saúde, 229 responderam aos instrumentos de coleta de dados, ou seja, 53,2\%, sendo 207 do gênero feminino e 22 do gênero masculino, representando, respectivamente, 90,4\% e 9,6\% da população estudada.

A maioria dos participantes trabalha 40 horas semanais, representando 76,0\%. É preocupante o fato de 13,5\% trabalharem mais de 70 horas semanais. Resultados semelhantes foram encontrados por Belancieri (2003) em estudo com trabalhadores da enfermagem de um hospital universitário.

Há que se discutir, ainda, a questão do duplo vínculo empregatício, pois 25,8\% dos participantes mantêm dois ou mais empregos, visando a complementar a renda familiar. De acordo com Belancieri (2003), isso pode comprometer a saúde e, consequentemente, a qualidade de vida do trabalhador da área da enfermagem.

Outro dado importante é a diferença salarial entre os gêneros. A remuneração das mulheres está numa faixa de $\mathrm{R} \$ 600,00$ a $\mathrm{R} \$ 1.000,00$ e a dos homens de $R \$ 1.100,00$ a $R \$ 1.500,00$. Esse dado é confirmado por Medeiros (2000), ressaltando que os salários das mulheres enfermeiras são 20\% menores em relação aos 230 homens enfermeiros.
A título de síntese, observa-se um encadeamento coerente dos dados no perfil sociodemográfico dos sujeitos em estudo, ou seja, a maioria está numa faixa etária mais madura (65\% entre 31 e 50 anos), é casada, com casa própria, com filhos, católica e com nível médio/técnico de escolaridade. A maioria é auxiliar de enfermagem e trabalha, no período diurno, há mais de 17 anos na enfermagem, com carga de 40 horas semanais, e apresenta um salário concentrado na faixa de $\mathrm{R} \$ 600,00$ a $\mathrm{R} \$ 1.500,00$.

\section{Níveis de resiliência dos trabalhadores da área da enfermagem}

Neste bloco de resultados são apresentados os níveis de resiliência, constituídos de sete fatores: regulação das emoções, controle dos impulsos, otimismo, análise causal, empatia, autoeficácia e exposição, em seus aspectos positivos e negativos.

De acordo com os resultados, a maioria dos participantes, em relação ao fator regulação de emoções, encontra-se abaixo da média (56,8\%), demonstrando que a capacidade de resiliência, nesse fator, encontra-se enfraquecida. No fator controle de impulsos, a maioria está acima da média (83,0\%). E nos fatores otimismo (79,5\%), análise causal (77,8\%), empatia (66,8\%), autoeficácia $(47,2 \%)$ e exposição $(51,5 \%)$, a maioria encontra-se na média (Tabela 1).

Reivich e Shatté (2002), autores do RQ-Test, utilizado nesta investigação, revelam que sujeitos a quem falta a habilidade de regular suas emoções apresentam dificuldades de construir e manter relacionamentos, especialmente, amizades. Existem provavelmente muitas razões para que isso ocorra. Delas, a mais básica é a negatividade. Quanto mais se associa à raiva, ao mau humor e à ansiedade, mais raivoso, mal-humorado e ansioso se torna o sujeito. Para ser resiliente, as emoções precisam ser expressas de maneira adequada, sejam elas positivas ou negativas.

O fator regulação das emoções pode estar relacionado às três categorias citadas por Trombeta e Guzzo (2002): condições do próprio indivíduo, condições familiares e condições relacionadas ao apoio/suporte do meio ambiente. No caso dos trabalhadores da área da enfermagem, embora não descartemos as características próprias do sujeito, o ambiente de trabalho, de 
Tabela 1. Resultado geral para os sete fatores de resiliência ( $n=229)$. Bauru (SP), 2008.

\begin{tabular}{|c|c|c|c|c|c|c|}
\hline \multirow{2}{*}{ Fatores } & \multicolumn{2}{|c|}{ Abaixo da média } & \multicolumn{2}{|c|}{ Média } & \multicolumn{2}{|c|}{ Acima da média } \\
\hline & $n$ & $\%$ & $n$ & $\%$ & $\mathrm{n}$ & $\%$ \\
\hline Regulação emoções & 130 & 56,8 & 93 & 40,6 & 6 & 2,6 \\
\hline Controle impulsos & 1 & 0,4 & 38 & 16,6 & 190 & 83,0 \\
\hline Otimismo & 20 & 8,7 & 182 & 79,5 & 27 & 11,8 \\
\hline Análise causal & 17 & 7,4 & 178 & 77,8 & 34 & 14,8 \\
\hline Empatia & 71 & 31,0 & 153 & 66,8 & 5 & 2,2 \\
\hline Autoeficácia & 78 & 34,0 & 108 & 47,2 & 43 & 18,8 \\
\hline Exposição & 73 & 31,9 & 118 & 51,5 & 38 & 16,6 \\
\hline
\end{tabular}

acordo com Vila (2005), parece ser a principal categoria relacionada ao estresse da profissão.

Para Reivich e Shatté (2002), resiliência não é apenas a capacidade de ultrapassar as adversidades, mas também capacitar-se a enfrentá-las e superá-las, melhorando os aspectos positivos da vida. Assim, a resiliência é a fonte para se alcançar os objetivos propostos.

A regulação das emoções e o controle dos impulsos estão intimamente relacionados; assim, os sujeitos que são fortes no fator controle dos impulsos tendem a ter alta regulação das emoções. Os autores ressaltam que esses dois fatores estão embasados em sistemas de crenças similares. Assim, quando o controle dos impulsos se apresenta abaixo da média, o sujeito aceita sua primeira crença impulsiva como verdadeira, e age de acordo com ela, produzindo, com frequência, consequências negativas que bloqueiam sua resiliência.

Diante das condições estressantes a que estão submetidos os trabalhadores da área da enfermagem, aliadas à dificuldade na regulação das emoções e do excessivo controle dos impulsos, pode-se ter como resultado uma redução da capacidade resiliente.

Dependendo da visão que o indivíduo tem da situação, de sua interpretação do evento gerador do estresse e do sentido a ele atribuído, que haverá ou não a condição de estresse. Acredita-se que há necessidade de discutir a resiliência não somente com os trabalhadores, mas também nas instituições, nos grupos e, especialmente, nos ambientes de saúde, visando a uma amplitude na atenção à saúde e às condições de trabalho.

Para Reivich e Shatté (2002), os sujeitos considerados resilientes são otimistas, uma vez que acreditam que as coisas podem sempre melhorar. Assumem o controle e a direção de suas vidas e têm esperança no futuro. Conforme centenas de estudos controlados, os otimistas são fisicamente mais saudáveis, têm menos probabilidade de sofrer de depressão e são mais produtivos no trabalho que os pessimistas.

Os autores demonstram em seus estudos que o otimismo e a autoeficácia, geralmente, caminham juntos, resultando na motivação para a busca de soluções às suas dificuldades. No trabalho, sujeitos otimistas, que acreditam em sua capacidade de resolver problemas, emergem como líderes.

O fator análise causal, segundo Reivich e Shatté (2002), refere-se à flexibilidade cognitiva das pessoas para identificar as causas de seus problemas. São realistas e não culpam outras pessoas por seus erros, visando preservar sua autoestima, bem como não gastam suas reservas ruminando sobre eventos ou circunstâncias que estão fora de seu controle.

Com fator empatia, Reivich e Shatté (2002) referem-se ao quanto uma pessoa é capaz de ler os indícios de estados emocionais de outras pessoas através da interpretação da linguagem não verbal (expressões faciais, tom de voz, linguagem corporal). Já outras pessoas que não desenvolveram essas habilidades são incapazes de se colocar no lugar dos outros. Essa incapacidade de ler as dicas não verbais pode dificultar as funções de gerenciamento, cujo trabalho compreende tanto a identificação de técnicas para motivação dos colaboradores como a valorização do outro.

No estudo realizado, a capacidade empática encontra-se na média, demonstrando que os trabalhadores da área da enfermagem são capazes de ler os indícios não verbais das outras pessoas, referentes a seus estados emocionais. 
O fator exposição refere-se à capacidade de expor-se, explorando seus verdadeiros limites, na busca de atenção e feedback de outras pessoas (Reivich \& Shatté, 2002); neste estudo, os sujeitos encontram-se dentro da média em relação a esse fator.

É interessante ressaltar que resultados semeIhantes foram encontrados em outros estudos. Embora somente o estudo de Belancieri (2007) tenha sido realizado com enfermeiras, considera-se importante citar também os estudos de Affonso (2007), desenvolvidos com educadores e adolescentes em liberdade assistida, a investigação de Barbosa (2006), com professores do ensino fundamental, e o estudo de Belancieri e Catharin (2007), com idosos do programa Universidade Aberta à Terceira ldade.

Belancieri e Cappo Bianco (2004), assim como Sória (2006), sugerem como estratégia para melhorar a capacidade resiliente dos trabalhadores da área da enfermagem, a reflexão do processo saúde-doença desde sua formação, expandindo para especialização e aprimoramentos, desenvolvendo, assim, suas habilidades internas necessárias para o fortalecimento da resiliência. Bianchini e Dell'aglio (2006) defendem mudanças nessas características internas e também nas externas, com o objetivo de incentivar o desenvolvimento de novas estratégias, mais eficazes, de enfrentamento das situações estressantes, através da promoção da resiliência no contexto da saúde.

Investigando sobre a resiliência na enfermagem e em outras áreas, Sória (2006) cita que estudos recentes demonstram uma lacuna em relação à utilização do conceito de resiliência na área da enfermagem na América Latina, fato que agrega valor à sua abordagem neste estudo.

A mesma autora demonstra ser interessante a instrumentalização das enfermeiras para a capacitação e formação de equipes a fim de que se possa identificar fatores de resiliência na clientela assistida de modo a colaborar na adesão ao plano de cuidados de enfermagem. No mundo contemporâneo, o conhecimento sistemático da resiliência é um instrumento válido e eficaz para todos os que se propõem a aperfeiçoar seu campo profissional, favorecendo o enfrentamento da 232 situação adversa.
Para finalizar, Belancieri e Cappo Bianco (2004) justificam essa necessidade ressaltando que o incentivo à promoção da resiliência e da saúde do profissional poderá contribuir para melhorar sua qualidade de vida, bem como reverter em melhor qualidade da assistência à saúde da população.

\section{Considerações Finais}

De acordo com os resultados obtidos nesse estudo, é possível tecer algumas considerações:

- A maioria dos trabalhadores da área da enfermagem é constituída de mulheres $(90,4 \%)$, corroborando o perfil em nosso País.

- Em relação aos níveis de resiliência, a maioria dos participantes apresenta uma discrepância entre os fatores regulação de emoções, que se encontra abaixo da média (56,8\%), e controle de impulsos, que está acima da média (83,0\%), o que pode resultar em elevado consumo de energia, prejudicando as atividades laborais. Nos fatores otimismo (79,5\%), análise causal (77,8\%), empatia (66,8\%), autoeficácia $(47,2 \%)$ e exposição (51,5\%), a maioria encontra-se dentro da média.

- Esses resultados podem estar associados ao perfil sociodemográfico, especialmente no que se refere à carga horária de trabalho e aos salários, sendo urgente o engajamento da categoria em movimentos, visando à aprovação do Projeto de Lei 2.295/2000, que tramita no congresso, a fim de aprovar e regulamentar a jornada de 30 horas semanais de trabalho na área da enfermagem.

- Outro aspecto que pode ser julgado interessante destacar é a resistência encontrada entre os trabalhadores da área da enfermagem em responder aos instrumentos de pesquisa, inclusive por parte dos responsáveis pela equipe. Embora se compreenda a rotina intensa de trabalho e a grande demanda de pacientes, entende-se que tal condição sugere o não envolvimento coletivo para a construção de uma categoria profissional mais forte, engajada nos novos rumos que a área profissional está tomando. Um aspecto que se acredita estar relacionado à melhoria das condições de trabalho e de saúde é o compromisso com o caráter acadêmico-científico na produção de conhecimentos que subsidiem a prática. 
- Nesse aspecto, discutir o caráter social e político da prática dos trabalhadores da área da enfermagem e refletir sobre a importância de sua participação nos rumos da própria profissão poderão minimizar o estado de alienação observado, uma vez que o papel desse profisisional não se resume ao saber técnico, mas apresenta também uma função social e política.

- Essas medidas, com certeza, serão revertidas na melhoria da qualidade de vida dos trabalhadores da área da enfermagem, bem como na melhoria da assistência à saúde dos usuários da Rede Municipal de Saúde.

\section{Referências}

Affonso, C. (2007). A liberdade assistida de adolescentes em cumprimento de medida socioeducativa e seus fatores de proteção: uma analise sob o olhar da psicologia sócio-histórica. Dissertação de mestrado não-publicada, Pontifícia Universidade Católica de São Paulo.

Barbosa, G. S. (2006). Resiliência em professores do ensino fundamental de $5^{a}$ a $8^{a}$ série: validação e aplicação do "Questionário do índice de Resiliência-adultos-Reivich-Shatté/Barbosa. Tese de doutorado não-publicada, Pontifícia Universidade Católica de São Paulo.

Belancieri, M. F. (2003). Estressee repercussões psicossomáticas em trabalhadores da enfermagem de um hospital universitário. Dissertação de mestrado não-publicada, Universidade do Sagrado Coração. Bauru.

Belancieri, M. F. (2005). Enfermagem: estresse e repercussões psicossomáticas. Bauru: EDUSC.

Belancieri, M. F. (2007) Promoção do processo de resiliência em enfermeiras: uma possibilidade. Tese de doutorado não-publicada, Pontifícia Universidade Católica de São Paulo.

Belancieri, M. F., \& Cappo Bianco, M. H. B. (2004). Estresse e repercussões psicossomáticas em trabalhadores da área da enfermagem. Revista Texto e Contexto de Enfermagem, 13 (1), 124-131.

Belancieri, M. F., \& Catharin, L. C. (2007). Resiliência e saúde na terceira idade. Anais do Fórum de Iniciação Científica da Universidade do Sagrado Coração. Bauru.

Bianchini, D. C. S, \& Dell'aglio, D. D. (2006). Processos de resiliência no contexto de hospitalização: um estudo de caso. Paidéia, 16 (35), 427-436.

Bulhões, I. (1994). Riscos do trabalho de enfermagem. Rio de Janeiro: Júlio C. Reis Livraria.

Flach, F. (1997). Resiliência: a arte de ser flexível. São Paulo: Saraiva.

Grotberg, E. H. (2005). Introdução: novas tendências em resiliência. In A. Mellilo \& E. N. Suárez-Ojeda (2005). Resiliência: descobrindo as próprias fortalezas (pp.15-22). Porto Alegre: Artmed.
Junqueira, M. F. P. S., \& Deslandes, S. F. (2003). Resiliência e maus tratos à criança. Cadernos de Saúde Pública, 19 (1) 227-235.

Lindstron, B. (2001). O Significado de resiliência. Revista Adolescente Latinoamericana, 3 (2), Porto Alegre.

Medeiros, S. M. (2000). As novas formas de organização do trabalho na terceira revolução industriale a força de trabalho em saúde: estudo em Natal-RN. Tese de doutorado nãopublicada, Universidade de São Paulo.

Moraes, M. C. L., \& Rabinovich, E. P. (1996). Resiliência: uma discussão introdutória. Revista Brasileira Crescimento e Desenvolvimento Humano, 6 (1/2), 10-13.

Nelson, R. (1997). Bounce back! Creating resilience from adversity. Toronto: Words Worth Professional Communications.

Pereira, A. M. S. (2001). Resiliência, personalidade, stress e estratégicas de coping. In J. Tavares. Resiliência e educação. São Paulo: Cortez.

Poletto, M., \& Koller, S. H. (2008). Contextos ecológicos: promotores de resiliência, fatores de risco e de proteção. Estudos de Psicologia (Campinas), 25 (3). Recuperado em abril 2008, disponível em: http://www.scielo.br/scielo. php? script=sci_arttext\&pid=S0103-166X200800030 0009\&lng=\&nrm =iso

Polk, L. V. (1997). Toward a middle-range theory of resilience. ANS. Advances in Nursing Science, 19 (3), 1-13.

Ralha-Simões, H. (2001). Resiliência e desenvolvimento pessoal. In J. Tavares (Org.), Resiliência e educação. São Paulo: Cortez.

Reivich, K., \& Shattè, A. (2002). The resilience factor: 7 essential skills for overcoming life's inevitable obstacles. New York: Broadway Books-Random House.

Sória, D. A. C. (2006). A resiliência dos profissionais de enfermagem na unidade de terapia intensiva. Tese de doutorado não-publicada, Universidade Federal do Rio de Janeiro.

Stacciarini, J. M. R., \& Tróccoli, B. T. (2001). O estresse na atividade ocupacional do enfermeiro. Revista Latino-Americana de Enfermagem, 9 (2), 17-25.

Trombetta, L. H. A. P., \& Guzzo, R. S. L. (2002). Enfrentando o cotidiano adverso: estudo sobre resiliência em adolescentes. Campinas: Alínea.

Vila, S. G. (2005). Qualidade de vida em enfermeiros de Bauru. Dissertação de mestrado não-publicada, Universidade do Sagrado Coração, Bauru.

Wollin, S. J., \& Wollin, S. (1993). The resilient self: how survivors of troubled families rise above adversity. New York: Villard Books.

Yunes, M. A. M. (2001). A questão triplamente controvertida da resiliência em familias de baixa renda. Tese de doutorado não-publicada, Pontifícia Universidade Católica de São Paulo.

Yunes, M. A. M., \& Szimanski, H. (2001). Resiliência: noção, conceitos afins e considerações críticas. In J. Tavares (Org.), Resiliência e educação. São Paulo: Cortez.

Recebido em: 27/4/2009

Versão final reapresentada em: 23/11/2009

Aprovado em: 19/2/2010 